\title{
Psicoterapias comportamentais no tratamento da depressão
}

\author{
Behavioral psychotherapy in the treatment of depression
}

\author{
Luciana Roberta Donola Cardoso
}

Mestre em Psicologia Experimental, psicóloga do Instituto de Psiquiatria do Hospital das Clínicas da Faculdade de Medicina da Universidade de São Paulo (USP), Vila Leopoldina, SP - Brasil, e-mail: lucidonola@uol.com.br

\section{Resumo}

A depressão é um dos transtornos psiquiátricos mais prevalentes no mundo. Estima-se que 5\% da população tem depressão. Tratamentos farmacológicos somados as psicoterapias têm mostrado resultados eficientes no tratamento da depressão. As psicoterapias comportamentais têm sido utilizadas no tratamento dessa patologia. Resultados como redução dos sintomas, aumento no repertório social e melhora nas relações sociais têm sido frequentemente associados a essas intervenções. O presente estudo bibliográfico objetivou descrever as intervenções comportamentais e suas aplicações no tratamento da depressão. As psicoterapias comportamentais aplicadas no tratamento da depressão encontradas na literatura foram: terapia comportamental ou analítico-comportamental; terapia da aceitação e compromisso; psicoterapia analítico funcional; ativação comportamental e terapia cognitivo-comportamental. De acordo com esta literatura, o indivíduo com depressão tende a apresentar redução na frequência de comportamentos reforçados positivamente (atividades prazerosas) e aumento, concomitante, de comportamentos de fuga e esquiva de situações aversivas. Parece que perda de reforçadores positivos e aumento de estímulos aversivos, bem como, dificuldade em lidar com situações-problema favoreceriam o desenvolvimento da depressão. O tratamento da depressão envolveria discriminação dos estímulos mantenedores desse transtorno, instalação e manutenção de comportamentos incompatíveis com os sintomas (queixa do cliente), aumento e/ou instalação de habilidades sociais, e aumento de atividades prazerosas. Um olhar global sobre esse fenômeno permite que o terapeuta identifique os déficits comportamentais que o indivíduo apresenta. Assim, modificar comportamentos e pensamentos parece ser importante quando se pretende tratar a depressão. As terapias "da aceitação e compromisso" e "ativação comportamental" apresentaram resultados significativos na prevenção do reaparecimento dos sintomas em longo prazo.

Palavras-chave: Depressão. Terapia comportamental. Tratamento. Revisão de literatura.

\begin{abstract}
Depression has been the psychiatric disorders more prevalent in the world. It is estimated that $5 \%$ of the population have depression. Pharmacological treatments combined psychotherapies have shown effective results in treating
\end{abstract}


depression. The behavioral psychotherapies have been used in the treatment of this pathology. Results such as reduction of symptoms, increased sociability and improvement in social relations have often been associated with these interventions. This review study aimed to describe the behavioral interventions and their applications in the treatment of depression. The applied behavioral psychotherapy in the treatment of depression in the literature was: behavior therapy or behavior analysis; therapy acceptance and commitment; functional analytic psychotherapy, behavioral activation and cognitive-behavioral. According to this literature, a person with depression tend to exhibit reduction in the frequency of positively reinforced behavior (pleasurable activities) and increased, concomitant behaviors of escape and avoidance of aversive situations. It appears that loss of positive reinforcement and increased aversive stimuli, as well as difficulty in dealing with problems would favor the development of depression. Treatment of depression involve discrimination of the stimuli maintainers of depression, installation and maintenance of behavior incompatible with the symptoms, increased and/or installation of social skills, and increase pleasant activities. A comprehensive global at this phenomenon allows the therapist to identify the behavioral deficits that the individual has. Thus, change behaviors and thoughts seem to be important when attempting to treat depression. Therapies "Therapy Acceptance and Commitment" and "Behavioral Activation" showed significant results in preventing the recurrence of symptoms in the long term.

Keywords: Depression. Behavioral therapy. Treatment. Review.

\section{Introduçáo}

Atualmente a depressão tem sido considerada um dos transtornos psiquiátricos mais prevalentes no mundo. De acordo com a Associação de Psiquiatria Americana (APA, 2004) estima-se que aproximadamente $5 \%$ da população geral apresentam depressão. Entretanto, em pacientes com outras comorbidades, como câncer e HIV, a prevalência de depressão pode chegar a 47\% (Fleck \& Horwath, 2005; Kessler et al., 2003).

A depressão é uma psicopatologia caracterizada por humor deprimido ou falta de motivação, perda de interesse ou prazer (anedonia) e cansaço ou fadiga. Além disso, alteração no peso corporal (ganho ou perda de peso significativo - 5\%), prejuízo no sono (insônia ou hipersonia), agitação ou retardo psicomotor, sentimento de inutilidade ou culpa, baixa capacidade de concentração ou tomada de decisão e/ou ideação suicida são outros sintomas relevantes no diagnóstico da depressão (APA, 2004; CID-10, 1993).

O episódio depressivo pode ser classificado como leve, moderado ou grave. Essa classificação varia de acordo com o número, gravidade e tempo de apresentação dos sintomas (APA, 2004).

$\mathrm{O}$ desenvolvimento ou aparecimento dos sintomas que caracterizam a depressão, comumente, está associado à presença de um evento aversivo, como, por exemplo, situações de estresse, descontentamento na realização de atividades cotidianas (trabalho, casa, família) e perda de algo ou alguém significativo para o indivíduo (morte de um ente querido, fim de um relacionamento, perda do emprego, aposentadoria, saída dos filhos de casa) (Dougher \& Hackbert, 2003).

Estudos longitudinais mostram que essa patologia pode ser crônica e recorrente. Sabe-se que mais da metade dos pacientes que receberam algum tipo de tratamento para depressão tiveram um segundo episódio ao longo da vida. As taxas de recidivas podem variar de $9 \%$ a $80 \%$ dependendo do tratamento (Antonuccio, Danton \& DeNelsky, 1995; Byrne, 2005; Kennedy et al., 2004; Byrne \& Rothschild, 1998).

Ensaios clínicos mostram que o tratamento farmacológico combinado com psicoterapia tem apresentado resultados mais eficientes na redução e prevenção do reaparecimento de episódios de depressão, do que aqueles apenas medicamentosos (Fleck \& Horwath, 2005; Hollon et al., 2005).

As psicoterapias comportamentais vêm apresentando resultados eficientes na modificação do comportamento de pessoas com depressão. Resultados como redução dos sintomas, aumento no repertório social e alteração na quantidade e qualidade das atividades e das interações sociais têm sido frequentemente associados a essas intervenções (Dougher, 1994; Dougher, 2000; Lewinsohn, Biglan \& Zeiss, 1976). 
O presente estudo bibliográfico objetivou descrever as intervenções comportamentais e suas aplicações no tratamento da depressão.

\section{Metodologia}

Foi realizada uma busca bibliográfica por meio de periódicos indexados nas bases de dados MEDLINE, SciELO e PsycINFO, cruzando os unitermos psychotherapists, behavioral therapy, cognitive-behavioral therapy, psicoterapias, terapia comportamental e terapia cognitivo-comportamental com os unitermos depressão e depression. Além disso, foram utilizados livros-texto de referência sobre o tema a fim de complementar a discussão sobre o assunto.

\section{Resultados e discussáo}

\section{Terapia comportamental ou analítico-comportamental}

Asintervenções baseadas na terapia comportamental visam a analisar funcionalmente os estímulos antecedentes e consequentes relacionados ao comportamento. Essa análise tem por objetivo identificar os eventos envolvidos no processo de aprendizagem do repertório comportamental do indivíduo (Abreu, 2006; Lewinsohn, Biglan \& Zeiss, 1976).

O terapeuta comportamental entende a depressão como uma redução na frequência de certas atividades tidas (anteriormente pelo indivíduo) como prazerosas e aumento, concomitante, de comportamentos de fuga e esquiva de situações aversivas. Esses comportamentos estariam relacionados à depressão em razão da dificuldade do indivíduo em acessar os reforçadores disponíveis em seu ambiente.

Segundo Ferster (1973), a redução na frequência de emissão de comportamentos consequenciados por reforçadores positivos poderia ser em decorrência do custo de resposta exigido em certas contingências de reforçamento e por mudanças repentinas no ambiente em que o indivíduo está inserido (Abreu \& Santos, 2008; Ferster, 1973).

Para Lewinsohn, Biglan \& Zeiss, (1976), além da mudança no ambiente em que a presença de reforçadores positivos não estariam mais disponíveis, a redução na taxa de resposta poderia ocorrer por uma perda no valor reforçador de determinados estímulos dispostos no ambiente (principalmente àqueles pareados aos estímulos contextuais) e/ou por uma alteração no repertório comportamental em que o indivíduo não conseguisse acessar os reforçadores disponíveis.

Já para Seligman (1975), a depressão estaria associada à perda de controle das contingências de reforçamento e a incapacidade de cessar a presença de estímulos aversivos. A incontrolabilidade, ou seja, incapacidade de eliminar a contingência aversiva favoreceria com que o indivíduo reduzisse a emissão de resposta.

Além disso, o indivíduo com depressão tende a ficar pouco atento a estímulos que sinalizam $\left(\mathrm{S}^{\mathrm{ds}}\right)$ as recompensas $\left(\mathrm{S}^{\mathrm{r}}\right)$ contingentes ao seu comportamento. Isso pode acarretar um atraso (aumento na latência) entre seu comportamento (resposta) e uma consequência recompensadora (por exemplo, receber atenção, carinho) (Abreu, 2006; Ferster, 1973).

$\mathrm{Na}$ terapia comportamental ou analítico-comportamental o tratamento é baseado na realização de uma análise funcional, ou seja, na descrição das variáveis (antecedentes e consequentes) relacionadas ao comportamento do indivíduo. Por meio dessa análise são identificados os comportamentos públicos (ações, falas) e encobertos (pensamentos, sentimentos) relacionados à instalação e manutenção do comportamento-problema. A identificação desses comportamentos permite que o terapeuta treine um repertório comportamental favorável para que o indivíduo possa obter reforçadores positivos no contexto em que está inserido (Abreu \& Santos, 2008; Jacobson \& Gortner, 2000).

Segundo Abreu (2006), para entender as alterações comportamentais que o cliente com depressão apresenta é necessário investigar as variáveis relacionadas à instalação e manutenção dos sentimentos de disforia ao longo de sua vida. Desse modo, seria possível fazer uma análise molar das contingências "depressoras", descrevendo os eventos antecedentes e consequentes aos comportamentos depressivos de interesse.

Outra variável importante no tratamento analítico-comportamental da depressão é favorecer com que o indivíduo reduza a exposição a ambientes aversivos (Mestre \& Hunziker, 1996). Ressalta-se que esses indivíduos podem apresentar autorregras bem instaladas relacionadas à impossibilidade de conseguir controlar, fugir ou se esquivar das situações aversivas (Hunziker, 2003). 
Intervenções que promovam o automonitoramento das atividades (por exemplo, utilização de diários) identificando os eventos que antecedem e sucedem seu comportamento poderiam possibilitar discriminações mais acuradas dessas situações e da maneira como o indivíduo reage a elas (Martell, Addis \& Jacobson, 2001; Rehm, 1977). Informações advindas do automonitoramento proporcionam ao terapeuta ferramentas para que possa planejar intervenções que promovam a extinção dos respondentes condicionados pareados a estas contingências aversivas. Aprendizagem ou desenvolvimento de do repertório discriminativo adequado permite que o indivíduo gradativamente volte a emitir comportamentos positivamente reforçados (Abreu, 2006).

Além disso, no tratamento da depressão o terapeuta deve promover um treinamento de habilidades sociais. O foco central desse treino é aumentar a exposição às situações sociais com o objetivo de restabelecer antigas fontes de reforçamento e possibilitar a instalação de novas fontes potenciais (Abreu \& Santos, 2008). A exposição às situações positivamente reforçadas seria incompatível com a emissão de comportamentos relacionados à depressão. A terapia teria função de auxiliar o indivíduo a se comportar de maneira mais funcional quando exposto aos eventos aversivos cotidianos. A exposição deve ser gradual considerando o grau de dificuldade na execução de cada tarefa. Sempre deve ser realizada pelas atividades em que o indivíduo relate menor grau de dificuldade.

\section{Terapia da aceitaçáo e do compromisso (ACT)}

A terapia da aceitação e do compromisso (ACT) tem por objetivo reduzir os comportamentos de esquiva. Essa terapia visa a interromper processos de aprendizagem decorrentes dos contextos socioverbais presentes em nossa comunidade. Esses processos tenderiam a levar à não aceitação e à resposta de esquiva a determinados sentimentos, como forma de resolução de problemas (Hayes \& Wilson, 1994; Hayes et al., 1999). Formulada a partir da proposta do distanciamento compreensivo, busca enfraquecer as esquivas emocionais e aumentar as respostas relacionadas à capacidade para mudança do comportamento, bem como "resgatar" reforçadores que atualmente não fazem parte do repertório do sujeito (Banaco et al., 2006; Brandão, 1999a).
As estratégias de intervenção da ACT são baseadas nos seguintes processos:

1) desamparo criativo: mostrar para o cliente que dentro do contex to no qual ele está inserido não existe solução e que a estratégia atual de resolução do problema é ineficaz (controle dos comportamentos encobertos). Assim, por meio da variabilidade comportamentalo cliente tende a desenvolver novas estratégias de resolução do problema;

2) controle doseventosprivados: descrever para o cliente as contingências relacionadas ao seu comportamento (encoberto) de esquiva;

3) discriminação entre o eu e o comportamento: promover a separação entre a pessoa que se comporta e o comportamento, criando um contexto em que se torne possível a aceitação de reações emocionais indesejáveis;

4) escolha e valorização de uma direção: alterar os comportamentos abertos em vez de controlar os encobertos;

5) abandono da disputa: aprender a aceitar os seus sentimentos. A luta do cliente com seus sentimentos são abandonados;

6) compromisso com a mudança: ao final busca-se uma redução na esquiva emocional e aumento das habilidades de assumir e manter compromisso de mudança comportamental (Brandão, 1999a).

Durante as sessões o terapeuta pode utilizar metáforas, paradoxos e exercícios experienciais a fim de ensinar o cliente a vivenciar os sentimentos, pensamentos, recordações e sensações. O uso dessas ferramentas tem por objetivo ensinar o cliente a contextualizar os eventos privados, clarificar o que almejam para suas vidas, identificar seus valores e prioridade e desenvolver um compromisso com as mudanças necessárias para modificar sua vida. Trabalhar valores é um aspecto importante dessa terapia (Brandão, 1999b; Hayes, Zettle \& Rosenfarb, 1989; Martell, Addis \& Jacobson, 2001).

Os comportamentos de esquiva apresentados por sujeito com depressão podem serobservados em dois momentos distintos. O primeiro ocorre durante o período de instalação dos comportamentos 
depressivos. O indivíduo recebe punições em seu ambiente contingentes a muitas classes de respostas. O segundo ocorreria após a instalação do repertório depressivo. Por exemplo, o indivíduo começa a faltar com frequência ao trabalho para evitar punições do chefe; aumenta ou diminui significativamente a ingestão de alimentos; começa dormir em excesso. A emissão desses comportamentos permite com que o indivíduo evite entrar em contato com os estímulos aversivos condicionados (Abreu, 2006; Martell, Addis \& Jacobson, 2001).

Alguns estudos vêm sendo desenvolvidos a fim de testar a eficácia da ACT no tratamento da depressão (Blackledge \& Hayes, 2006; Forman et al., 2008; Hayes \& Smith, 2005; Zettle \& Hayes, 1986). Estudos longitudinais mostraram que pacientes com depressão submetidos à ACT, comparados com aqueles que não receberam nenhuma intervenção e com aqueles submetidos às intervenções cognitivas, apresentaram melhora significativa em relação à redução nos comportamentos de esquiva dos sentimentos relacionados ao problema, aumento na aceitação do problema e nos comportamentos relacionados à autoconfiança. Observou-se que após seis meses essas alterações comportamentais se mantiveram (Blackledge \& Hayes, 2006; Forman et al., 2008; Zettle \& Hayes, 1986).

Ainda comparando os resultados da terapia da aceitação e compromisso com outras abordagens cognitivas, estudos mostraram que os pacientes submetidos à ACT apresentaram redução mais rápida nos pensamentos disfuncionais (autodepreciação e pensamentos negativos) do que os abordados por terapias cognitivas convencionais (Hayes, Follette \& Linehan, 2004; Hayes, Luoma, Bond, Masuda \& Lillis, 2006; Zettle \& Rains, 1989).

\section{Psicoterapia analítica funcional (FAP)}

A Psicoterapia Analítica Funcional (FAP) tem por objetivo analisar a interação cliente/terapeuta no ambiente terapêutico como base para modificação de comportamentos clinicamente relevantes (Kohlenberg \& Tsai, 1994; Kohlenberg \& Tsai, 2001; Vandenberghe \& Pereira, 2005).

Os comportamentos clinicamente relevantes (CRB) são analisados por meio de três tipos. Os CRB1, que são os comportamentos relacionados ao problema que ocorrem durante a sessão e que devem ser modificados; e os CRB2, que são os comportamentos considerados adequados para obtenção da melhora do cliente que ocorrem durante a sessão. Vale ressaltar que os CRB2 só aparecem quando os CRB1 foram alterados. Há também os CRB3, que representam as crenças e interpretações do cliente acerca do seu problema (Kohlenberg \& Tsai, 1994).

Desse modo, durante as sessões o componente terapêutico central é promover um arranjo de contingências relacionado ao problema no contexto terapêutico e modificar os comportamentos emitidos durante as sessões para que o cliente possa generalizar essas respostas fora do contexto terapêutico (Banaco et al., 2006).

Para promover a mudança do comportamento do cliente, o terapeuta estrutura as sessões por meio de cinco regras. Essas regras consistem em:

1) observara emissão dos comportamentos clinicamente relevantes (CRBs);

2) evocar;

3) e reforçar os CRBs;

4) observaros efeitos potencialmente reforçadores do comportamento em relação aos CRBs do cliente;

5) fornecer interpretações das variáveis que afetam o comportamento do cliente (Vandenberghe, 2009).

No caso do tratamento da depressão, inicialmente o terapeuta observa e registra as queixas do cliente relacionadas ao problema (por exemplo: desânimo, incompreensão, apatia, anedonia, remorso sobres situações do passado e dificuldade para fazer novos contatos em situações sociais). Essas queixas seriam os CRB1. Em seguida, o terapeuta, por meio das cinco regras supracitadas, treina, pela modelagem e modelação, as habilidades sociais, manejo da raiva, enfrentamento de situações de estresse e exposições a situações sociais. Por fim, o terapeuta observa e registra as mudanças comportamentais promovidas com a intervenção (Kohlenberg \& Tsai, 2001; Vandenberghe, 2009).

Com essa estrutura de intervenção, tem-se observado resultados significativos no tratamento da depressão. Alterações relacionadas à melhora nos relacionamentos sociais, diminuição do número de queixas, retorno às atividades que foram deixadas de serem praticadas por conta da depressão, aumento de relatos de prática de atividades descritas 
como prazerosas, planos sobre o futuro, aceitação de mudanças de planos e adequação de estratégias para realização dos planos estão entre os resultados obtidos com essa intervenção (García, Aguayo \& Montero, 2006).

Cordova e Kohlenberg (1994) observaram que a promoção da auto-observação e aceitação de mudanças comportamentais (CRB3) reduziu significativamente as respostas emocionais que evocavam sentimentos de culpa durante a sessão. A exposição dessas respostas emocionais durante as sessões e o direcionamento para que o paciente pudesse observar e entender a maneira como reage diante desses estímulos favoreceram a modificação dos comportamentos de depressão.

Assim, na psicoterapia analítico funcional a descrição e a alteração das contingências relacionadas aos comportamentos depressivos do cliente durante as sessões proporcionariam as alterações comportamentais. Acessar os comportamentos-problema durante a sessão permite modelar um novo repertório comportamental, incompatível com os comportamentos relacionados à instalação e manutenção da depressão (Dougher \& Hackbert, 1994; Kohlenberg, Hayes \& Tsai, 1993; Naugle \& Follette, 1998). Assim, a efetividade dessa terapia no tratamento da depressão estaria atrelada à generalização das alterações comportamentais promovidas durante a terapia para fora do contexto terapêutico em situações semelhantes (García, Aguayo \& Montero, 2006; Biglan, 1991).

\section{Ativaçáo comportamental (behavior activation - BA)}

A ativação comportamental tem por objetivo aumentar o repertório comportamental e promover atividades de resolução de problemas para que o indivíduo possa entrar em contato com as contingências de reforçamento positivo (Jacobson et al., 1996).

Nessa intervenção, o terapeuta deve identificar as contingências que estão mantendo os comportamentos depressivos do cliente e alterá-las. $\mathrm{Na} \mathrm{BA}$ o terapeuta conduz análises funcionais dos comportamentos-problema do cliente, bem como incentiva e ensina o indivíduo a fazê-la. Nessas análises o terapeuta fica atento aos comportamentos clinicamente significativos associados à instalação $\mathrm{e}$ manutenção do problema (Abreu, 2006; Gortner, Gollan, Dobson \& Jacobson, 1998; Kanter, Cautilli, Bush \& Baruch, 2005).

Por meio da análise funcional o terapeuta deve sinalizar ao indivíduo como seus comportamentos depressivos estão sendo consequenciados; descrever as consequências a curto, médio e longo prazo da maneira como estálidando com o problema; e apontar a ineficácia da estratégia na resolução deste (Dunner, 2004; Martell, Addis \& Jacobson, 2001).

Além das análises funcionais, comumente o terapeuta se utiliza das seguintes técnicas: diário com enfoque na descrição do progresso; registro das atividades; avaliação das atividades por meio dos critérios: "domínio" e "prazer", manejo de contingências situacionais, ensaio comportamental, modelagem de estratégias de ativação e ensaio verbal das tarefas propostas. O termo "domínio" se refere a sentimentos de gratificação durante o desempenho de uma tarefa específica e o termo "prazer" refere-se a sentimentos de apreciação, entretenimento ou diversão a partir de uma dada atividade (Abreu \& Santos, 2008; Beck, Rush, Shaw \& Emory, 1979)

Jacobson et al. (1996), em um estudo longitudinal, observaram que os pacientes com depressão maior submetidos à ativação comportamental apresentaram melhores resultados quanto à redução dos sintomas de depressão e manutenção da mudança comportamental nos dois anos seguintes, comparados com aqueles que receberam outras intervenções cognitivas e cognitivo-comportamentais. Nesse estudo as sessões foram estruturadas para identificar os problemas cotidianos específicos, propor atividades semiestruturadas aos sujeitos e treinar comportamentos que aumentariam a probabilidade do contato do indivíduo com reforçadores naturais do ambiente. Além disso, os pacientes eram encorajados a monitorar, por meio de um diário, suas atividades, descrevendo o grau de "domínio e prazer" para cada uma delas. Ao final de cada sessão os terapeutas discutiam com os participantes as situações-problema e descreviam as contingências em que poderiam obter reforçadores positivos.

Outro estudo comparou o tratamento farmacológico (paroxetina), tratamento com placebo, terapia cognitivo-comportamental e ativação comportamental em uma amostra de 500 sujeitos diagnosticados com depressão. Nesse estudo, os participantes submetidos à ativação comportamental tinham que atribuir uma nota hierárquica, de acordo 
com o grau de dificuldade, para as atividades que gostariam de realizar. Toda vez que os indivíduos conseguiam realizar alguma das atividades descritas na lista eram recompensados por uma ficha que mais tarde era trocada por algo que gostavam (prêmios previamente estabelecidos). Os participantes foram avaliados antes e depois da intervenção por meio do Inventário de Depressão Beck (BDI). Aqueles submetidos ao BA apresentaram resultados significativamente melhores quanto à redução dos sintomas de depressão do que aqueles submetidos à outra terapia comportamental. Os resultados para esses sujeitos foram semelhantes àqueles submetidos ao tratamento farmacológico (paroxetina). Além disso, os sujeitos com sintomas mais graves da doença que foram submetidos ao tratamento com BA apresentaram redução dos sintomas significativamente superiores aos que foram submetidos à terapia cognitivo-comportamental (Dimidjian et al., 2004). A taxa de sintomas recorrentes durante os dois anos de acompanhamento foram menores para aqueles submetidos à ativação comportamental, do que para aqueles submetidos ao tratamento medicamento e à TCC (Dobson et al., 2004).

\section{Terapia cognitivo-comportamental (TCC)}

A terapia cognitivo-comportamental consiste em desenvolver e aplicar estratégias que favoreçam mudanças comportamentais e cognitivas. A escolha das estratégias utilizadas é determinada de acordo com os comportamentos que se pretende alterar (Ito, 1998; Knapp \& Beck, 2008; Shinohara, 2001).

As intervenções cognitivo-comportamentais são baseadas em ensinar o paciente a identificar e lidar com as situações relacionadas aos sintomas da doença, bem como treinar repertório de habilidades sociais e resolução de problemas. O objetivo central dessa intervenção é alterar excessos ou déficits comportamentais, crenças e valores relacionados aos comportamentos-problema (Knapp \& Beck, 2008; Powell, Abreu, Oliveira \& Sudak, 2008).

As técnicas e estratégias comportamentais mais utilizadas no tratamento da depressão são aquelas relacionadas ao automonitoramento (diários), reestruturação cognitiva, técnicas de enfrentamento (iniciar ou realizar alguma atividade), treino de habilidades sociais e resolução de problemas. Essas estratégias têm por objetivo ensinar o indivíduo a identificar sentimentos e pensamentos automáticos disfuncionais que estão relacionados ao seu sentimento de disforia. Registrar o grau de emoção para cada atividade registrada proporciona um autoconhecimento sobre seus sentimentos, valores e crenças (Beck, Rush \& Emory, 1997; De Oliveira, 2008; Powell et al., 2008). O paciente deve registrar tanto atividades prazerosas e produtivas quanto aquelas que lhe trazem tristeza ou desânimo em realizá-las. As atividades que ajudam o paciente a desenvolver um autoconhecimento e monitoramento do seu comportamento permitem com que ele entre em contato com as contingências que podem estar mantendo o problema.

As aplicações dessas técnicas muitas vezes são estruturadas em protocolos de atendimento. Esses protocolos tendem a delimitar o que será abordado com o paciente a cada sessão. Para o tratamento da depressão, o número de sessões pode variar de 12 a 16 atendimentos.

A terapia cognitivo-comportamental tem mostrado resultados significativos no tratamento da depressão, tanto na redução dos sintomas quanto na prevenção do reaparecimento do problema, comparada com outras intervenções breves e com tratamentos medicamentosos (Dobson, 1989; Hollon et al., 2005; Robinson, Berman \& Neimeyerm 1990; Teasdale et al., 2000).

Estudo de intervenção mostrou que adolescentes com depressão maior tratados com fluoxetina e TCC durante 12 semanas apresentaram resultados significativamente superiores em relação à remissão dos sintomas se comparados com aqueles tratados apenas com o medicamento. Além disso, estudos de revisão mostraram que os pacientes tratados com TCC apresentaram resposta de melhora em relação à remissão dos sintomas significativamente mais duradoura do que os pacientes tratados com medicações (Bockting et al., 2008; Greenberg \& Fisher, 1997).

\section{Conclusão}

A depressão tem sido considerada um dos transtornos psiquiátricos mais prevalentes no mundo. Graças à alta prevalência, profissionais da área da saúde têm se interessado cada vez mais em conhecer intervenções que mostram resultados significativos na redução dos sintomas e na prevenção do 
reaparecimento do problema. Neste estudo de revisão foram observados diversos trabalhos que utilizam as psicoterapias comportamentais no tratamento da depressão. Dentre elas estão a terapia comportamental ou analítico-comportamental; terapia da aceitação e compromisso; psicoterapia analítico funcional; ativação comportamental e terapia cognitivo-comportamental. A aproximação dessas terapias se dá na maneira como o fenômeno, no caso a depressão, é observado, descrito e analisado. De acordo com essa literatura, o indivíduo com depressão tende a apresentar redução na frequência de comportamentos positivamente reforçados (atividades prazerosas) e aumento, concomitante, de comportamentos de fuga e esquiva de situações aversivas. Parece que a perda de reforçadores positivos e aumento da presença de estímulos aversivos, bem como dificuldade em lidar com situações-problema favoreceriam o desenvolvimento da depressão. Um olhar global sobre o fenômeno permite que o terapeuta identifique os déficits comportamentais que o indivíduo apresenta.

Baseado nessas intervenções, o tratamento da depressão envolveria discriminação dos estímulos mantenedores do problema, instalação e manutenção de comportamentos incompatíveis com os sintomas (queixa do cliente), aumento e/ou instalação de habilidades sociais e, principalmente, aumento de atividades prazerosas. Além disso, parece importante treinar o enfrentamento das situações aversivas para a resolução dos problemas. Assim, modificar comportamentos e pensamentos relacionados ao problema parece ser importante quando se pretende tratar essa patologia.

As terapias denominadas "terapia da aceitação e compromisso" e "ativação comportamental" mostraram resultados significativos na prevenção do reaparecimento dos sintomas em longo prazo.

Vale ressaltar que neste estudo observou-se que terapeutas comportamentais não excluem um tratamento combinado com medicação (psicoterapia e antidepressivos). Sabe-se que um tratamento multidisciplinar tem mostrado resultados eficientes na redução dos sintomas de depressão.

\section{Referências}

Abreu, P. R. (2006). Terapia analítico-comportamental da depressão: Uma antiga ou uma nova ciência aplicada? Revista de Psiquiatria Clínica, 33(6), 322-328.
Abreu, P. R., \& Santos, C. (2008). Behavioral models of depression: A critique of the emphasis on positive reinforcement. International Journal of Behavioral and Consultation Therapy, 4, 130-145.

American Psychiatric Association (2004). Manual diagnóstico e estatístico de transtornos mentais-DSMIV-TR. (Dayse Batista Trans.). Porto Alegre: Artmed. (Publicado originalmente em 2000).

Antonuccio, D. O., Danton, W. G., \& DeNelsky, G. Y. (1995). Psychotherapy versus medication for depression: Challenging the conventional wisdom with data. Professional Psychology: Research \& Practice, 26(6), 574-585.

Banaco, R. A., Cardoso, L. R. D., Matos, D. C., Menezes, M. S. T. B., Pasquinelli, R. H., \& Souza, M. R. (2006). Proposta de práticas clínicas: Um estudo exploratório. In H. J. Guilhardi, N. C. de Aguirre. (Org.). Sobre comportamento e cognição: Expondo a variabilidade (pp. 371-381). Santo André: ESETec.

Beck, A. T., Rush, A. J., Shaw, B. F., \& Emory, G. (1979). Cognitive therapy of depression. New York: Guilford.

Beck, A. T., Rush, A. J., Shaw, F. B., \& Emery, E. (1997). Terapia cognitiva da depressão. Porto Alegre: Artes Médicas.

Biglan, A. (1991). Distressed behavior and its context. The Behavior Analyst, 13, 157-169.

Blackledge, J . T., \& Hayes, S. C. (2006). Using acceptance and commitment training in the support of parents of children diagnosed with autism. Child \& Family Behavior Therapy, 28(1), 1-18.

Bockting, C., Ten Doesschate, M. C., Spijker,J., Spinhoven, P., Koeter, M. W., \& Schene, A. H. (2008). Continuation and maintenance use of antidepressants in recurrent depression. Psychother Psychosom, 77(1), 17-26.

Brandão, M. Z. S. (1999a). Terapia comportamental e análise funcional da relação terapêutica: Estratégias clínicas para lidar com o comportamento de esquiva. Revista Brasileira de Terapia Comportamental e Cognitiva, 1(2), 179-187.

Brandão, M. Z. S. (1999b). Abordagem contextual na clínica: Revisão da ACT e proposta de atendimento. In R. A. Ranaco (Org.). Sobre o comportamento e cognição: Aspectos teóricos, metodológicos e de formação em análise do comportamento e terapia cognitiva (pp. 148-155). São Paulo: ESETec. 
Byrne, S. E., \& Rothschild, A. J. (1998). Loss of antidepressant efficacy during maintenance therapy: Possible mechanisms and treatments. Journal of Clinical Psychiatry, 59(6), 279-288.

Byrne, B. M. (2005). Factor analytic models: Viewing the structure of an assessment instrument from three perspectives. Journal of Personality Assessment, 85(1), 17-32.

Classificação de transtornos mentais e de comportamento da CID-10. (1993). Descrição e diretrizes diagnósticas. Porto Alegre: Artes Médicas.

Cordova, J. V., \& Kohlenberg, R. J. (1994). Acceptance and the therapeutic relationship. In S. C. Hayes, N. S. Jacobson, V. M. Follette, \& M. J. Dougher (Org.). Acceptance and change: Content and context in psychotherapy (pp. 125-140). Reno: Context Press.

De Oliveira, I. R. (2008). Trial-Based Thought Record (TBTR): Preliminary data on a strategy to deal with core beliefs by combining sentence reversion and the use of analogy with a judicial process. Revista Brasileira de Psiquiatria, 30(1), 12-18.

Dimidjian, S. A., Hollon, S. D., Dobson, K. S., Schmaling, K. B., Kohlenberg, R. J., \& Rizvi, S., et al. (2004). Methods and acute-phase outcomes. In S. D. Hollon (Org.). Behavioral activation, cognitive therapy, and antidepressant medication in the treatment of major depression. Symposium conducted at the annual meeting of the American Psychiatry Association, New York.

Dobson, K. S., Hollon, S. D., Dimidjian, S. A., Schmaling, K. B., Kohlenberg, R. J., \& Rizvi, S., et al. (2004). Prevention relapse. In S. D. Hollon, (Org.). Behavioral activation, cognitive therapy, and antidepressant medication in the treatment of major depression. New York: Symposium conducted at the annual meeting of the American Psychiatry Association.

Dobson, K. S. (1989). A meta-analysis of the efficacy of cognitive therapy for depression. Journal of Consulting and Clinical Psychology, 57(3), 414-419.

Dougher, M. J. (1994). Introduction. The Behavior Analyst, 17, 287-288.

Dougher, M. J. (2000). Clinical behavior analysis. Reno: Context Press.

Dougher, M. J., \& Hackbert, L. (1994). A behavioranalytic account of depression and a case report using acceptance-based procedures. The Behavior Analyst, 17, 321-334.
Dougher, M. J., \& Hackbert, J. A. (2003). Uma explicação analítico-comportamental da depressão e o relato de um caso utilizando procedimentos baseados na aceitação. Revista Brasileira de Terapia Comportamental e Cognitiva, 5(2), 167-184.

Dunner, D. L. (2004). Methods and acute-phase outcomes. In S. D. Hollon (Org.). Behavioral activation, cognitive therapy, and antidepressant medication in the treatment of major depression (pp. 143-148). In Symposium conducted at the annual meeting of the American Psychiatry Association, New York.

Ferster, C. B. (1973). A functional analysis of depression. American Psychologist, 28, 857-870.

Fleck, M. P., \& Horwath, E. (2005). Pharmacologic management of difficult-to treat depression in clinical practice. Psychiatr Serv, 56, 8, 1005-1011.

Forman, E. M., Hoffman, K. L., McGrath, K. B., Herbert, J. D., Bradsma, L. L., \& Lowe, M. R. (2008). Terapia de aceitação e compromisso: Modelo, dados e extensão para a prevenção do suicídio. Revista Brasileira Terapia de Aceitação e Compromisso, 10(1), 81-104.

García, R. F., Aguayo, L. V., \& Montero, M. C. (2006). Application of functional analytic psychotherapy: Clinical analysis of a patient with depressive disorder. The Behavior Analyst Today, 7(1), 1-18.

Gortner, E. T., Gollan, J. K., Dobson, K. S., \& Jacobson, N. S. (1998). Cognitive-behavioral treatment fordepression: Relapse prevention. Journal of Consulting and Clinical Psychology, 66(2), 377-384.

Greenberg, R. P., \& Fisher, S. (1997). Mood mending medicines: Probing drug, psychotherapy and placebo solutions. In S. Fisher, \& R. P. Greenberg (Org.). From placebo to panacea: Putting psychiatric drugs to the test (pp. 115-172). New York: John Wiley \& Sons.

Hayes, S. C., Zettle, R. D., \& Rosenfarb, I. (1989). Rule -Following. In S. C. Hayes (Org.). Rule-governed behavior: Cognition, contingencies, and instructional control (pp.153-190). New York: Plenum Press.

Hayes, J. A., \& Wilson, K. G. (1994). Acceptance and commitment therapy. The Behavior Analyst, 17: 289-303.

Hayes, S. C., Bissett, R. T., Korn, Z., Zettle, R. D., Rosenfarb, I. S., Cooper, L. D., et al. (1999). The impact of acceptance versus control rationales on pain tolerance. The Psychological Record, 49, 33-48. 
Hayes, S. C., \& Smith, S. (2005). Get out of your mind and into your life: The new acceptance and commitment therapy. Oakland, CA: New Harbinger.

Hayes, S. C., Follette, V. M., \& Linehan, M. M. (2004). Mindfulness and acceptance: Expanding the cognitive behavioral tradition. New York: Guilford Press.

Hayes, S. C., Luoma, J., Bond, F., Masuda, A. \& Lillis, J. (2006). Acceptance and commitment therapy: Model, processes, and outcomes. Behaviour Research and Therapy, 44(1), 1-25.

Hollon, S. D., Shelton, R. C., \& Loosen, P. T. (1991). Cognitive therapy and pharmacotherapy for depression. Journal of Consulting and Clinical Psychology, 59(1), 88-99.

Hollon, S. D., Jarrett, R. B., Nierenberg, A. A., Thase, M. E., Trivedi, M., \& Rush, A. J. (2005). Psychotherapy and medication in the treatment of adult and geriatric depression: Which monotherapy or combined treatment? Archives of General Psychiatry, 66(4), 455-468.

Hunziker, M. H. L. (2003). Desamparo aprendido. Tese de livre-docência, Instituto de Psicologia, Universidade de São Paulo, São Paulo.

Ito, L. M. (1998). Terapia cognitivo-comportamental para transtornos psiquiátricos. Porto Alegre: Artes Médicas.

Jacobson, N. S., Dobson, K., Truax, P. A., Addis, M. E., Koerner, K., Gollan, J. K., et al. (1996). A component analysis of cognitive-behavioral treatment for depression. Journal of Consulting and Clinical Psychology, 64(2), 295-304.

Jacobson, N. S., \& Gortner, E. (2000). Can depression be de-medicalized in the 21 st century: Scientific revolutions, counter-revolutions and magnetic field of normal science. Behavior Research and Therapy, 38(2),103-117.

Kanter, J. W., Cautilli, J. D., Bush, A. M., \& Baruch, D. E. (2005). Toward comprehensive functional analysis of depressive behavior: Five environment factors and a possible sixth and seventh. The Behavior Analyst Today, 6(1), 65-85.

Kennedy, S. H., Lam, R. W., Nutt, D. J., \& Thase, M. E. (2004). Psychotherapies, alone and in combination. In S. H., Kennedy, R. W. Lam, D. J. Nutt., \& M. E. Thase (Org.). Treating depression effectively: Applying clinical guidelines. London: Martin Dunitz.
Kessler, R. C., Berglund, P., Demler, O., Jin, R., Koretz, D., Merikangas, K. R., et al. (2003). National Comorbidity Survey Replication. The epidemiology of major depressive disorder: Results from the National. Comorbidity Survey Replication (NCS-R). JAMA, 289(23), 3095-3105.

Knapp, P., \& Beck, A. T. (2008). Fundamentos, modelos conceituais, aplicações e pesquisa da terapia cognitiva. Revista Brasileira de Psiquiatria, 30(2), S54-64.

Kohlenberg, R. J., Hayes, S. C., \& Tsai, M. (1993). Radical behavioral psychotherapy: Two contemporary examples. Clinical Psychology Review, 13(6), 579-592.

Kohlenberg, R. J., \& Tsai, M. (1994). Improving cognitive therapy for depression with functional analytic psychotherapy: Theory and case study. The Behavior Analyst, 17, 305-319.

Kohlenberg, R. J., \& Tsai, M. (2001). Psicoterapia analítica funcional: Criando relações terapêuticas intensas e curativas. Santo André: ESEtec.

Lewinsohn, P. M., Biglan, A., \& Zeiss, A. S. (1976). Behavioral treatment of depression, In Davidson, P. O. (Org.). The behavioral management of anxiety, depression and pain. New York: Brunner/Mazel.

Martell, C. R., Addis, M. E., \& Jacobson, N. S. (2001). Depression in context: Strategies for guided action. New York: W. W. Norton.

Mestre, M. B. A., \& Hunziker, M. H. L. (1996). O desamparo aprendido em ratos adultos, como função de experiências aversivas incontroláveis na infância. Tuiuti: Ciência e Cultura, 6(2): 25-47.

Naugle, A. E., \& Follette, W. C. (1998). A Functional Analysis of Trauma Symptoms. In W. C. Follette, J. I. Ruzek, \& F. R. Abueg (Org.). Cognitive-behavioral therapies for trauma. New York: Guilford.

Powell, V. B., Abreu, N., Oliveira, I. R., \& Sudak, D. (2008). Terapia cognitivo-comportamental da depressão. Revista Brasileira de Psiquiatria, 30(Sup 2), S73-80.

Rehm, L. P. (1977). A self-control model of depression. Behavior Therapy, 8, 787-804.

Robinson, L. A., Berman, J. S., \& Neimeyer, R. A. (1990). Psychotherapy for the treatment of depression: A comprehensive review of controlled outcome research. Psychol Bull, 108(1), 30-49.

Seligman, M.E. P. (1975). Helplessness: On depression, development and death. San Francisco: Freeman. 
Shinohara, H. O. (2001). Conceituação da terapia cognitivo-comportamental. In R. A. Ranaco (Org.). Sobre o comportamento e cognição: Aspectos teóricos, metodológicos e de formação em análise do comportamento e terapia cognitiva (pp. 18-21). São Paulo: ESETec.

Teasdale, J. D., Segal, Z. V., Williams, J. M., Ridgeway, V. A., Soulsby, J. M., \& Lau, M. A. (2000). Prevention of relapse/recurrence in major depression by mindfulness-based cognitive therapy. Journal of Consulting and Clinical Psychology, 68(4), 615-623.

Vandenberghe, L., \& Pereira, M. B. (2005). O papel da intimidade na relação terapêutica: uma revisão teórica à luz da análise clínica do comportamento. Psicologia: Teoria e Prática, 7(1), 127-136.

Vandenberghe, L. (2009). Keeping the focus on clinically relevant behavior: Supervision for functional analytic psychotherapy. International Journal of Behavioral and Consultation Therapy, 5(2), 209-222.

Zettle, R. D., \& Hayes, S. C. (1986). Dysfunctional control by client verbal behavior. The context of reason-giving. Analysis of Verbal Behavior, 4, 30-38.

Zettle, R. D., \& Rains, J. C. (1989). Group cognitive and contextual therapies in treatment of depression. Journal of Clinical Psychology, 45(3), 436-445.

Recebido: 09/03/2010

Received: 03/09/2010

Aprovado: 19/05/2010

Approved: 05/19/2010 